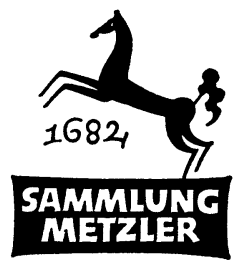

REALIENBÜCHER FÜR GERMANISTEN ABT. D: LITERATURGESCHICHTE 


\title{
GOTTFRIED \\ VON STRASSBURG
}

\author{
VON \\ GOTTFRIED WEBER \\ IN VERBINDUNG MIT \\ WERNER HOFFMANN
}

MCMLXII

J.B.METZLERSCHE VERLAGSBUCHHANDLUNG

STUTTGART 
ISBN 978-3-476-99268-0

ISBN 978-3-476-99267-3 (eBook)

DOI 10.1007/978-3-476-99267-3

C 1962 Springer-Verlag GmbH Deutschland

Ursprünglich erschienen bei J. B. Metzlersche Verlagsbuchhandlung und Carl Ernst Poeschel Verlag GmbH in Stuttgart 1962. 


\section{VORWORT}

Anlage und Inhalt des vorliegenden Bandes sind durch die Zielsetzung der /Sammlung Metzler / bestimmt: sein Zweck ist die Vermittlung von Realien und Fakten. Deren gibt es freilich im Falle Gottfrieds von Straßburg nicht allzu viele - dafür um so mehr der offenen Fragen, der voneinander abweichenden Ansichten, der einander widerstreitenden Deutungen. Diese Forschungssituation gerade galt es aufzuzeigen und die verschiedenen Auffassungen zu skizzieren. $\mathrm{Daß}$ dies mit dem Bemühen um unbedingte Sachlichkeit geschehen ist, dürfte, so hoffe ich, deutlich geworden sein.

Was meine eigene >Tristan «-Untersuchung (1953) anlangt, so sind die wesentlichsten Stellungnahmen zu ihr im Literaturverzeichnis angeführt. Für eine kritische Auseinandersetzung ist hier nicht der Ort; eine solche bleibt einer in Vorbereitung begriffenen eigenen Auseinandersetzung vorbehalten,

Auch bei dem vorliegenden Bändchen der > Sammlung Metzler` ging es darum, den akademischen Unterricht zu fördern und zu entlasten sowie eine Grundlage für weitere Erkenntnisse darzubieten.

Frankfurt am Main, im Januar 1962 GotTfried Weber 


\section{ABKÜRZUNGSVERZEICHNIS}

ADB Allgemeine Deutsche Biographie

AfdA Anzeiger für deutsches Altertum und deutsche Literatur

Beitr. Beiträge zur Geschichte der deutschen Sprache und Literatur

BSB Sitzungsberichte der Akademie der Wissenschaften zu Berlin, philologisch-historische Klasse

DLZ Deutsche Literaturzeitung

DPh. Deutsche Philologie im Aufriß

DVjs. Deutsche Vierteljahrsschrift für Literaturwissenschaft und Geistesgeschichte

DU Der Deutschunterricht (Stuttgart)

Et. Germ. Etudes Germaniques

Euph. Euphorion

GR

The Germanic Review

GRM Germanisch-Romanische Monatsschrift

Hist. Jb. Historisches Jahrbuch

IF Indogermanische Forschungen

Kürschners Deutsche National-Litteratur. Historisch kritische Aus-

DNL gabe, hrsg. von Joseph Kürschner

Med. Aev. Medium Aevum

mhd

mittelhochdeutsch

MLQ

Modern Language Quarterly

MLR

MSB

The Modern Language Review

Sitzungsberichte der Akademie der Wissenschaften zu München, philosoph.-philolog. und histor. Klasse

Neophil. Neophilologus

nhd neuhochdeutsch

NZZ Neue Zürcher Zeitung

RG Revue Germanique

Triv. Trivium

Vf.-Lex. Die deutsche Literatur des Mittelalters. Verfasserlexikon

WaG Die Welt als Geschichte

WW Wirkendes Wort

ZfdA Zeitschrift für deutsches Altertum und deutsche Literatur

ZfDk. Zeitschrift für Deutschkunde

ZfdPh. Zeitschrift für deutsche Philologie

ZföG Zeitschrift für die österreichischen Gymnasien 


\section{IN HALT}

I. Kapitel: Gottfrieds Lebensverhältnisse . . . . . . . I

II. Kapitel: Gottfrieds Dichtungen . . . . . . . . 7

I. Die Gottfried zugeschriebenen lyrischen Dichtungen........... 7

2. Die Uberlieferung von Gottfrieds ITristan . . Io

3. Zur Entstehung (Datierung) des >Tristan ८. . . I4

4. Zu Gottfrieds Sprach- und Verskunst . . . 2 I

III. Kapitel: Der Tristanstoff in der mittelalterlichen Dichtung 25

IV. Kapitel: Forschungsgeschichte, Forschungsstand,

Forschungsaufgaben . . . . . . . . 44

Register . . . . . . . . . . . . . 84 